\title{
Les victimes silencieuses des crises humanitaires et l'(in)sécurité de leurs activités économiques: Etude de cas parmi les migrants de deux villes tchadiennes
}

\author{
Syntyche Nakar Djindil \\ Wageningen University, Law and Governance Group \\ The Netherlands \\ syntyche.djindilnakar@wur.nl \\ Mirjam de Bruijn \\ Africa Studies Centre, Leiden \\ The Netherlands \\ bruijnm@ascleiden.nl
}

\section{RÉSUMÉ}

Lorsqu'une catastrophe humanitaire fait la une des médias, la communauté internationale se mobilise à réduire les conséquences les plus sérieuses. La population du Tchad connaît cependant des crises endémiques qui ne sont liées à aucun élément déclencheur particulier ; elle ne reçoit pas l'assistance internationale nécessaire pour faire face à ces problèmes. La présente étude concerne 111 ménages de migrants de la région du centre du Tchad, qui ont, à cause de la guerre et de la sécheresse, tout perdu, et qui vivent désormais dans de N’Djamena et de Mongo ; ces familles sont confrontées à la précarité et à d'autres éléments qui menacent leurs moyens de subsistance. Des méthodes qualitatives et quantitatives ont été combinées dans l'étude pour révéler l'histoire intrigante de leur vie quotidienne au milieu de crises complexes et endémiques. Des données anthropométriques et sanitaires ont été utilisées pour déterminer l'état nutritionnel des mères et de leurs enfants de moins de cinq ans. Des narrations de vie, des entretiens en profondeur et des observations participatives ont permis aux chercheurs de déterminer les stratégies de négociation que ces familles adoptent pour accéder à leur nourriture et à leurs abris, leur expérience de l'insécurité alimentaire et de la vulnérabilité sanitaire, ainsi que les conséquences que ces éléments ont sur leur vie quotidienne. Les résultats indiquent que $62 \%$ des ménages sont dirigés par des femmes, qu’il existe de forts taux de malnutrition aiguë (40-50\%) et chronique (35-40\%) et que 46\% des mères sont sous-alimentées et anémiées. Les taux de mortalité infantile se sont avérés également élevés : de 30\% à 42\%. 97\% des enfants n’ont pas été complètement vaccinés, voire pas vaccinés du tout, dans certains cas. Aucun des ménages n'a accès à l'eau potable et aux services sociaux de base. La corruption endémique et l'abus manifesté par les autorités tchadiennes ont été identifiés comme sources d'insécurité quotidienne. Ces migrants considèrent cette situation misérable comme normale.

\section{MOTS-CLEFS}

Migrations urbaines, victimes silencieuses, crise humanitaire, activités de subsistance, Tchad. 
En 1984, la sécheresse et surtout les activités des rebelles ont ruiné ma famille, ainsi que les réserves et les cultures de notre village. Nous n'avions rien à manger et le futur du village était bien sombre. Il ne pleuvait pas, et le sol était très sec... J'ai donc décidé de quitter le village pour me rendre à Mongo, pour trouver de la nourriture : mon but était alors de revenir sous peu. Lorsque je suis arrivé à Mongo, la situation était terrible. De nombreuses personnes étaient arrivées de différents villages en raison de linsécurité et de la faim... J'aurais pu attendre un mois pour être enregistré en tant que réfugié, vivre dans un camp et recevoir de la nourriture tous les jours. Ce n'était pas mon but, et ce nétait à mon avis pas une solution. Je devais trouver un moyen de nourrir ma famille... Je ne pouvais pas non plus revenir au village les mains vides, donc j’ai décidé de poursuivre mon chemin ; jai pris la route de N'Djamena. La première année, c'était difficile. Je vivais dans les rues de Djamalbar avec d'autres personnes pauvres. Des soldats parcouraient les rues dans la nuit, arrêtaient des hommes et les envoyaient combattre, donc nous devions trouver un moyen de nous cacher lorsque les $4 \times 4$ des soldats passaient. C'était une période diffcile, mais quelques mois plus tard, j'ai trouvé un emploi de coursier. C'était difficile de gagner même 1000 FCFA transporter dix sacs de $100 \mathrm{~kg}$ par jour (chaque sac me donnait 100 FCFA). Avec l'argent, j’ai acheté ce terrain. A l'époque, ce n'était pas cher, et j'ai pu y faire venir ma famille du village.

-Hassan, 44 ans, Nguéli, un quartier marginal de N’Djamena, janvier 2005.

\section{Introduction}

Comme Hassan, de nombreuses victimes des crises au Tchad doivent gagner leur vie en négociant les moyens de subsistance face aux menaces et à l'incertitude. On trouve ces personnes partout : dans les villes et dans les villages. Ces personnes sont confrontées à des crises similaires à celles qui ont de manière générale frappé l'Afrique Centrale lors des quarante dernières années : guerre, sécheresse et insécurité économique. Ces crises conditionnent la vie quotidienne de nombreuses personnes au Tchad qui demeurent invisibles, qui ne disposent d'aucune possibilité pour exprimer leurs inquiétudes et qui n'ont, en fin de compte, pas le choix. La survie dans de telles circonstances devient pénible, puisque ces personnes doivent trouver un certain équilibre entre insécurité et incertitude chaque jour, et doivent déterminer s'ils seront en mesure de nourrir leur famille.

Ces histoires devraient se situer dans le contexte régional de l'Afrique Centrale, qui a été le théâtre de crises de nature et d'origine diverses, et qui sont désormais endémiques et chroniques. C'est le cas du Tchad, où la guerre et l'insécurité, les sécheresses, la dégradation écologique, la famine et les épidémies ont entaché l'histoire et la vie de la population. Depuis les années soixante, le ays connaît l'instabilité. La cohésion sociale demeure fragile, et les conflits armés sont imminents. La gouvernance politique, économique et institutionnelle est faible, et dans la plupart des cas corrompue. Enfin, l'insécurité et l'oppression politique con- 
tinuent de freiner le développement de la société civile (Azevedo, 1998; Miles, 1995; De Bruijn et Van Dijk, 2007a; De Bruijn et Van Dijk, 2008; Van Dijk, 2007; International Crisis Group, 2006). Les conditions de vie sont précaires, et peuvent se dégrader à tout moment. Par conséquent le Tchad est régulièrement classé parmi les dix pays les moins développés au monde, selon l'Indice de Développement Humain (Rapports sur le Développement Humain, PNUD, 1990-2008). En dépit de la récente production pétrolière, qui a permis d'accroître le PNB et d'autres indicateurs économiques, les indicateurs sociaux et sanitaires se dégradent continuellement. La malnutrition infantile et les taux de mortalité sont toujours élevés ; l'accès aux services sociaux, à l'eau potable et à l'assainissement, est médiocre, et le nombre des ménages dirigés par les femmes est en augmentation en raison des conflits, des épidémies et de la migration due à l'emploi. (WGI, 2008; OECD, 2007; Djindil et al., 2007; USAID, 2008). Des générations ont été et continueront d'être affectées dans leurs capacité physique, moral et intellectuel et, sont condamnées à naître, vivre et donner naissance dans ce contexte de vulnérabilité, d'insécurité et de mauvaise gouvernance.

En dehors de la guerre civile de 1979, les sécheresses des années 80 et la récente crise du Darfour qui ont attiré une importante aide humanitaire internationale, la plupart des tchadiens connaissent des crises endémiques qui ne sont pas nécessairement liées à des facteurs déclencheur spécifique et pour cela, ne reçoivent pas l'attention internationale. Le reste du monde sait peu de choses à propos des " catastrophes silencieuses " au Tchad. De récentes recherches ont permis de mieux comprendre les multiples expériences de la guerre civile dans les zones urbaines et rurales du Tchad connues pour leur insécurité et instabilité persistantes (De Bruijn et Van Dijk, 2007b; Dickow, 2005; Eriksson et Hagströmer, 2005). Ces études sont importantes pour comprendre les aspects historiques et politiques des crises généralisées au Tchad ; cependant, elles ne fournissent pas des informations sur les conditions de vie des victimes silencieuses. Nous tenterons dans cet article de retracer la vie de ces personnes en analysant la sécurité des activités génératrices de leurs revenus dans des environnements urbains tchadiens. La sécurité de ces activités est basée sur le degré d'accès aux ressources pour la survie et comprend les aspects physiques, écologiques, moraux, socioéconomiques, culturels et politiques d'une quantité minimum d'éléments nécessaires à la durabilité de telles activités. Les ménages pauvres et vulnérables en marge de la société tchadienne connaissent une incertitude et une insécurité extrêmes, et doivent s'engager dans des " manœuvres " interpersonnelles et communautaire pour créer et maintenir ces activités.

Les activités économiques des individus sont basées sur la quantité et la qualité des ressources auxquelles ils ont accès. Ces ressources peuvent être de divers types (social, économique, physique, naturel, humain) et peuvent être privées ou publiques. Les ménages ont des niveaux différents d'accès à ces ressources, ce qui affecte leur capacité à prévoir et à faire face aux chocs. La menace de l'insécurité alimentaire des individus influence également leurs activités (Chambers et Conway, 1992; Devereux, 2001).

Pour comprendre leur position, nous avons choisi, pour cette étude, de combiner des méthodes de recherche qualitatives et quantitatives. L'analyse et la méthodologie qualitatives permettent de narrer des articles qui offrent une perspective dans la négociation des ressources pour la 
survie de ces «victimes» (par exemple De Bruijn et Djindil, 2006 ou De Bruijn, 2007 sur les enfants de la rue à N'Djamena). La littérature dans ce domaine montre que, dans des circonstances défavorables, les individus développent leur propre vie de manière créative (Essed et al., 2005). Cet article se penche sur les individus qui ont fuit les crises et ont déménagé dans une ville où ils gagnent leur vie dans la périphérie de cette dernière. La capacité des individus démunis à négocier l'accès aux ressources, équipements et réclamations (c'est à dire la capacité à concrétiser les choses auxquelles ils ont droit) détermine le niveau de la sécurité de leurs activités économiques (Ribot et Peluso, 2003; De Bruijn, 2007; De Bruijn et Djindil, 2006). Notre étude analyse les conditions de vie et les expériences des individus qui ont migré vers deux grandes villes : Mongo, la capitale de la région de Guera, et N'Djamena, la capitale du Tchad, située à $500 \mathrm{~km}$ à l'ouest. Nous avons en particulier examiné la manière dont ces personnes négocient l'accès aux besoins de première nécessité (santé, habitat, nourriture, eau potable) tout en faisant face à l'insécurité chronique et à de hauts niveaux de stress. Une telle compréhension pourrait en effet permettre de définir une assistance appropriée dans une situation de crise endémique et, enfin, d'améliorer l'efficacité à long terme des programmes d'assistance humanitaire. La déstabilisation de la sécurité des activités économiques peut affecter négativement la sécurité alimentaire des ménages, l'état physique et psychologique des individus, et le développement des capacités physiques et mentales de leurs enfants. Ces aspects ont été saisis en mesurant le statut nutritionnel et enregistrant l'histoire de vie des familles qui ont participé à l'étude.

\section{Méthodologie}

\section{Travail sur le terrain}

De novembre 2002 à avril 2003, des données anthropométriques, sociologiques et anthropologiques ont été collectées auprès des migrants de la région de Guera, qui vivent dans les banlieues de N'Djamena et dans le Secteur 4 de Mongo. A N’Djamena, la plupart des migrants vivent dans des quartiers périphériques où ils pouvaient accéder à des terrains inoccupés. En première étape, nous avons exploré certains de ces quartiers afin d'identifier les migrants de la région de Guera et d'évaluer la faisabilité de futures recherches dans leur communauté. Il n'a pas été simple d'établir un contact : la méfiance, les abus verbaux et un refus quasi-systématique exprimé par la plupart des personnes interrogées ont clairement montré qu'une telle perspective ne serait pas possible. Néanmoins, les habitants de deux quartiers, Diguel Zafaye et Nguéli - ont manifesté une volonté de répondre à notre demande. Là, nous avons rencontré respectivement 33 et 30 ménages provenant des mêmes villages, et qui souhaitaient collaborer avec notre équipe de recherche et nous aider à établir des contacts supplémentaires au sein de leur communauté. A Mongo, la plupart des migrants vivent dans le Secteur 4, où nous avons travaillé avec 48 ménages d'ethnies différentes.

\section{Insécurité alimentaire et activités économiques}

Pour aborder notre sujet principal, qui était la caractérisation des stratégies relatives aux moyens de subsistance, nous avons mené une série d'entretiens sur les histoires de vie avec des hommes et des femmes, individuellement et en groupe. Les discussions portaient sur leurs expéri- 
ences durant diverses crises avant leur migration et les stratégies qu'ils avaient employé au cours de la migration pour se loger et se nourrir. Certaines personnes, surtout des hommes, ont refusé de parler de leurs expériences de la guerre et des problèmes liés à la politique nationale, reflétant un climat général de crainte et de traumatisme. Cependant, ils ont été en mesure de nous expliquer les stratégies et tactiques utilisées pour se construire une nouvelle vie. Les femmes se sont avérées plus ouvertes et ont fourni des récits plus détaillés.

La perturbation des relations économiques, culturelles et sociales peuvent déstabiliser l'accès quantitatif et qualitatif à la nourriture, ce qui affecte l'état physique et psychologique des adultes et le développement des capacités mentales et physiques de leurs enfants. Ces aspects ont été saisis en mesurant l'état nutritionnel des mères et de leurs enfants de moins de cinq ans, et en enregistrant l'histoire de vie de leurs familles. Pour estimer le niveau d'insécurité alimentaire des ménages, notre travail empirique s'est penché sur deux grandes questions : la disponibilité de la nourriture et les possibilités liées à l'accès aux aliments. Pour les enfants de moins de cinq ans, nous nous sommes également intéressés aux méthodes d'allaitement et de sevrage. "La sécurité alimentaire existe lorsque tous les individus, à tout moment, disposent d'un accès physique et économique à une alimentation nutritive, suffisante et saine qui satisfait leurs besoins diététiques et leurs préférences alimentaires pour une vie active et saine " (FAO, 1996). Cette définition intègre la stabilité, l'accès à la nourriture, la disponibilité d'aliments suffisamment nutritifs et l'utilisation biologique de la nourriture.

Des données socioéconomiques et démographiques ont été collectées en plus des estimations de base relatives à l'économie des ménages. Les facteurs potentiels de vulnérabilité et d'inégalité ont également été analysés : état civil, niveau d'éducation, profession, nombre d'enfants et de membres dépendants, la principale source de revenus, l'accès aux soins médicaux, à la scolarité et à l'eau potable, ainsi que la participation aux réseaux sociaux.

\section{Situation sanitaire et nutritive des enfants et de leurs mères}

Les femmes et les enfants sont le groupe le plus vulnérable parmi les migrants. Nous avons utilisé des questionnaires structurés pour obtenir des informations démographiques, nutritionnelles et sanitaires des enfants et de leurs mères.

Dans tous les ménages enquêtés, nous avons prélevé des mesures anthropométriques (poids, taille, périmètre brachial) des enfants âgés de 6 à 59 mois et de leurs mères. Nous avons de plus mesuré le taux d'hémoglobine de chaque mère. Par manque de documents d’état civile, l’âge de chaque enfant a été fourni par la mère en fonction du calendrier local établi à l'occasion. Pour chaque enfant, les indices Z-score de poids/taille qui exprime l'émaciation, et de taille/âge qui exprime le retard de la croissance, ont été calculés par le logiciel Epi-info et interprétés conformément aux normes de NCHS (National Centre for Health Statistics population), adoptées par l'OMS et le CDC comme bases internationales de mesure de l'état nutritionnel des enfants. Toute valeur de Z-score inférieure à -2 est considérée comme en dessous de la normalité, et en dessous de -3, la malnutrition est considérée comme sévère (OMS, 1995). La classification de l'état nutritionnel des mères a été faite en fonction de l'Indice de Masse Corporelle (IMC) qui est le rapport du poids en $\mathrm{Kg}$ par la taille en mètre au carré. Selon les normes, 
il y a déficit énergétique lorsque l'IMC $<18,5 \mathrm{Kg} / \mathrm{m}^{2}$ et obésité quand l'IMC est $>25 \mathrm{Kg} / \mathrm{m}^{2}$. Le taux d'hémoglobine a été mesuré avec le système Hémo-Cue et interprété conformément aux seuils de OMS comme suit : lorsque le taux d'hémoglobine est compris entre 11,9 et $10 \mathrm{~g} / \mathrm{dL}$, l'anémie est légère, elle est dite modérée quand le taux de $\mathrm{Hb}$ est compris entre 9,9 et 7 et sévère quand la valeur de $\mathrm{Hb}$ est inférieure à $7 \mathrm{~g} / \mathrm{dL}$.

Les principaux résultats de l'étude sont présentés dans les sections qui suivent ; une introduction est proposée pour chaque partie, suivie d'illustration des aspects relatifs ál'implication du contexte social spécifique sur les moyens de subsistance et enfin les conséquences au niveau des ménages sont décrites á travers le statut nutritionnel et sanitaire des mères et des enfants, les perturbations sociales (réduction de la dynamique des individus, acquisition de la nourriture) et la souffrance psychologique en rapport avec le contexte social. L'insécurité alimentaire et la vulnérabilité sont manifestes dans l'état sanitaire et nutritionnel des mères et des enfants, dans les troubles sociaux liés aux historiques de vie et enfin dans la détresse psychologique des personnes interrogées.

\section{Les crises au Tchad : étude des périphéries urbaines}

Avant d'aborder les études de cas, il convient d'expliquer ce que nos entretiens ont révélé à propos de la complexité des crises que vivent les habitants du Tchad. La juxtaposition et l'analyse des divers points de vue des migrants ont permis une reconstruction progressive de l'historique de la guerre et les sécheresses au Tchad. Parmi les 111 chefs de ménages de notre échantillon, 73\% avaient quitté leur village entre les années 1970 et 1990. Ces décennies ont été marquées par trois grands événements au Tchad : les sécheresses sahéliennes de 1970 et 1980, la guerre civile de 1979 et tous les troubles qui précédaient l’accession de Hissein Habré au pouvoir en 1982 et celle de Idriss Deby en 1990 (crises transitionnelles supplémentaires et répression exercée par Hissein Habré sur des communautés d'Hadjaray en 1987).

Après la révolte des paysans de 1965 à Mangalmé et des rébellions similaires au milieu des années 1990, différents groupes de rebelles ont choisi des zones spécifiques de la région de Guera pour recruter des soldats et mettre en place des repaires stratégiques dans les montagnes. Les rebelles, ou "bandits ", comme les appellent les habitants locaux, ont provoqué d'immenses problèmes socioéconomiques et des dommages psychologiques dans les villages. Les villageois se sont retrouvés au milieu du gouvernement et des rebelles, qui les ont tous deux accusé de complicité. La dégradation des conditions de vie dans ces villages a contraint de nombreux habitants à abandonner leurs domiciles. Pour des raisons logistiques et sécuritaires, la Croix Rouge a déplacé de nombreuses personnes vers les centres urbains pour trouver de la nourriture et bénéficier de soins médicaux ; la plupart n'est jamais retournée au village. La migration est une réaction à l'insécurité et à la violence par les individus qui vivent sous pression politique et environnementale. Gallais a décrit la mobilité comme une "condition sahélienne " typique, mais au Tchad, ce ne sont pas seulement ces conditions sahéliennes qui poussent les individus à migrer : il s'agit également de l'insécurité (Gallais, 1975). La présence de tant de Hadjaray à $\mathrm{N}^{\prime}$ Djamena est une réaction à l'insécurité et à diverses formes de violence. Comme beaucoup l'ont indiqué, les sécheresses sont un phénomène naturel au Sahel auquel les populations sont habituées, mais la violence des rebelles et les autres menaces exigent différentes stratégies de 
gestion. Dans de telles circonstances, les crises de routine considérées comme "normales " deviennent insupportables et l'ultime solution est alors de partir. Comment les individus survivent-ils cependant lorsqu'ils partent ? Notre recherche à N'Djamena et à Mongo révèle une partie de cette histoire.

\section{Diguel Hadjaray : un quartier de la banlieue de N'Djamena}

Je suis le chef des Hadjaray à Diguel Zafaye. Dans mon village, jétais cuisinier pour les Soeurs de l'Eglise Catholique. Après 1976, les attaques rebelles sont devenues fréquentes : les sours étaient menacées, et la situation n'a fait qu'empirer. Les rebelles brutalisaient tout le village. Ils ont forcé les sours à quitter la région de Guera et à aller à N'Djamena. C'est comme cela que je suis arrivé à N'Djamena en 1978. J'ai vécu avec les sours, et plus tard, ma femme et mes enfants $m$ 'avaient rejoint. Je voulais retourner au village, mais ce n'était pas possible. Dans ma région, et partout au Tchad, les mouvements rebelles devenaient plus violents, et la guerre civile a commencé à N'Djamena. Les soeurs sont reparties dans leur pays (la France) et j'ai dî chercher un autre emploi pour nourrir ma famille. Cela fait neuf ans que je vis ici. Tout va bien et j'espère que cela va continuer ainsi. Depuis que j'ai quitté le village, c'est la première fois que je vis aussi longtemps dans un endroit fixe. Avant cela, je vivais dans les quartiers de Kabalaye, Ardeb-Djoumal et Sabangali. J'étais comme un nomade, et mon logement dépendait du type d'emploi que j'avais. La plupart de mes compatriotes connaissent une vie de nomade.

Souleyman Azarak, Diguel, mai 2003

Situé au nord de la ville, Diguel est l'un des plus grands quartiers de N'Djamena. Le quartier est divisé en carrés, qui portent les noms des groupes ethniques ou des activités de leurs habitants. Diguel Zafaye, où nous avons mené notre étude, est située à la périphérie; nous avons pu rencontrer différents groupes ethniques de la région du Guera, que l'on appelle Hadjaray (signifiant rocher en arabe et en référence au terrain montagneux du Guera). Les individus, pour la plupart des Dangaliat de Korlongo, ont été forcés de quitter leurs villages pour diverses raisons (guerre, attaques rebelles, sécheresse, famine ou conflit). Souleyman, le chef des Hadjaray à Diguel Zafaye, est un Dangaliat ; c'est lui qui a pu trouver comment accéder à des espaces inoccupés à Diguel. Le nom Diguel Hadjaray a été officiellement reconnu en 1994 par le service des Cadastres. 33 ménages de l'ethnie Dangaliat dont 15 dirigés par des femmes avaient participé à notre étude.

L'âge moyen auquel les chefs de ces ménages étaient venus à N'Djaména est de dix-neuf ans : $6 \%$ étaient arrivés avant 1980, 76\% entre 1980 et 1990, et $18 \%$ entre 1990 et 2003 . La majorité $(80 \%)$ confirme avoir quitté le village pour deux raisons primordiales : les sécheresses et les attaques fréquentes des rebelles qui leur avaient dérobé tous leurs biens et ne favorisaient pas un climat serein pour le travail agricole, qui pourtant constituait leur seule source de survie.

Jusqu'en 1990, l'occupation des terrains à Diguel Zafaye était arbitraire et pratiquement libre. Parfois, le chef de quartier autorisait les individus à utiliser certaines parcelles pour leur logement ou leurs activités agricoles en échange de petites sommes d'argent. Souleyman Azarak (voir ci-dessus) est le premier Dangaliat à avoir trouvé un terrain à Diguel Zafaye ; il a alors informé les autres Dangaliat de la ville des possibilités qui y existaient. Dans notre échantillon, 
27 ménages avaient reçu cette information de lui, et avaient saisi l'opportunité d'accéder aux terrains et de s'installer à Diguel, échappant ainsi aux pressions de la ville. En 1994, le service des cadastres a initié d'expulser tous les habitants sans documentation officielle certifiant sa propriété et ceux qui ne seraient pas en mesure de payer pour régulariser leur terrain. Le marché foncier autour de Diguel s'est développé, tout comme l'abus des autorités publiques envers les habitants. Souleyman Azarak poursuit son récit, lors de notre entretien, sur la création de Diguel Hadjaray :

En 1994, le service des cadastres a noté une forte concentration d'Hadjaray dans cette partie du quartier de Diguel, ainsi le nom "Diguel Hadjaray " fut accordé à l'espace que nous occupons. Je suis né en 1951 à Korlongo dans la région de Guera. J'ai sept enfants. Lorsque je suis arrivé à N'Djamena, jétais comme un nomade. Je n'avais pas de logement stable, et ce dernier dépendait de mon travail. Après avoir perdu mon travail avec les sours, j’ai été garde, mais les gens ne macceptaient pas comme garde, car j'ai une famille nombreuse, et mon salaire n'était pas suffisant pour louer une maison. Par conséquent, j'ai décidé de trouver un endroit en dehors de la ville. J'ai cherché pendant des mois, et enfin, un de mes amis m'a indiqué qu'il y avait une possibilité en bordure de Diguel. J'ai commencé par un jardin, et plus tard, j'ai construit une maison, et y ai fait venir ma famille. Maintenant, la communauté Hadjaray de Diguel est grande, et offre une certaine sécurité. Le jardin est lucratif, mais exige beaucoup d'investissement : le sol est de mauvaise qualité, et l'eau est un sérieux problème. Maintenant, j'ai une pompe motorisée, mais la quantité d'eau dépend de la pluie et des gens de l'autre côté qui utilisent parfois la même source.

En dépit des conditions difficiles à N'Djamena (rareté des terrains, faible fertilité des sols et faibles précipitations), les Hadjaray ont pu recréer la vie d'un village à Diguel Zafaye. Le travail agricole reste la source principale de revenus pour la majorité. L'influence urbaine sur leur style de vie se traduit par le fait que les femmes peuvent se lancer dans du petit commerce (sucre, thé, gombo, etc.) et que les hommes peuvent trouver des emplois manuels. Au niveau physique également, Diguel Zafaye est un peu comme un village : toutes les maisons sont construites en terre battue, et les champs se trouvent aux alentours des concessions.

Les ménages qui comptent en moyenne quatre personnes sont considérés comme pauvres, comme l'indiquent les conditions sanitaires et nutritionnelles des mères et de leurs enfants : la boule de céréale est leur plat principal et est accompagnée de sauces différentes - en général du gombo : la consommation de produits laitiers et de protéines est rare. La fréquence des repas quotidiens varie entre un et deux, et seuls 13 ménages mangent régulièrement deux repas par jour. Vingt-deux des 36 enfants âgés entre six et cinquante-neuf mois, souffrent de malnutrition aiguë. Dix-sept de ces enfants souffrent de malnutrition chronique, ce qui est le produit d'une alimentation inappropriée sur une longue période. Il n’y a pas de méthode de sevrage appropriée pour les enfants, qui, aussitôt sevrés, doivent partager les repas avec les adultes. Seuls sept enfants sont complètement immunisés. Vingt-neuf femmes souffrent d'une anémie dont seize sous une forme sévère avec des taux d'hémoglobine en dessous de $7 \mathrm{~g} / \mathrm{dL}$. Ces résultats correspondent au déficit énergétique des mères. Seules neuf des trente-trois femmes ont un IMC normal $\left(18,5-25 \mathrm{~kg} / \mathrm{m}^{2}\right)$, et le reste $(\mathrm{n}=24)$ pèse moins que le poids normal. 
Ces indicateurs illustrent le niveau de souffrance physique. La mortalité infantile est assez élevée, avec deux morts toutes les cinq naissances. Ceci pourrait être lié aux problèmes rencontrés durant la grossesse, au faible poids des enfants à la naissance, à un sevrage inapproprié et en particulier à la sous-alimentation. Seuls six ménages ont confirmé qu'ils disposaient de ressources suffisantes pour s'alimenter correctement ; parmi ceux-ci, quatre possèdent un jardin et des arrosoirs à motopompes leur permettant de produire des légumes à vendre durant l'année. Sept femmes métayères sont plus vulnérables, et leur faible niveau de productivité ne leur permet pas de payer le propriétaire des terres qu'elles cultivent.

Depuis 2002, Diguel est en expansion et de nombreux bâtiments neufs sont actuellement érigés. En conséquence, il existe désormais des installations privées pour l'eau : ceci permet aux habitants d'avoir un accès temporaire à de l'eau potable. Ces activités de construction à Diguel ont permis aux hommes d'obtenir des emplois manuels et des possibilités de création de petites entreprises (vente de beignets, de noisettes, de sucre, de thé) pour les femmes. Ces activités contribuent à la sécurité alimentaire de 21 ménages, mais malheureusement, cette situation ne durera que peu de temps.

L'expansion de la ville menace les personnes qui ne disposent pas de papiers en règle pour les terrains qu'elles occupent. La régularisation des frontières reste un sérieux problème. Les expulsions sont toujours imminentes, et cela augmente la crainte parmi les migrants. Souleyman Azarak poursuit son récit :

Bien sur, je suis le chef des Hadjaray, mais il est difficile de gérer tous leurs problèmes. Les problèmes fonciers sont en particulier sensibles et politisés. Je dois être prudent et stratège pour protéger mes propriétés et mes relations sociales. Il existe des hommes riches qui souhaitent de plus en plus acquérir des terrains dans le quartier, et ils tentent de simposer par tous les moyens. Ça minquiète beaucoup. Certains de mes compatriotes m'accusent d'être leurs complices et d'aider les riches et le service des Cadastres, mais Dieu seul sait sous quelle pression je vis. Je me pose des questions tous les jours quant à mes relations futures avec mes compatriotes

La scolarité est au-delà des moyens financiers de $97 \%$ des ménages et l'accès aux soins médicaux est également problématique. Le centre sanitaire le plus proche est à peu près $5 \mathrm{~km}$; le coût d'une consultation est entre 200 FCFA et 500 FCFA (ce qui équivaut à $1 \mathrm{~kg}$ de riz), et la qualité du service est parfois douteuse. Le rapport qualité / prix est insignifiant, ce qui explique que les femmes se sont organisées pour faire les accouchements à domicile en s'appuyant sur l'aide des femmes âgées parmi elles. Les femmes ainsi que les hommes sont plutôt favorables aux soins traditionnels et aux réseaux des " choukous " (guérisseurs traditionnels). La réaction d'Halima Kinger, qui vit à Diguel Zafaye, citée ci-dessous, illustre le manque de confiance locale quant au centre sanitaire de N'Djamena :

Je ne vais plus à l'hôpital, et je ne conseille à personne d'y aller. Lorsque j'étais au village, je pensais que la vie en ville était meilleure, et que les soins médicaux y étaient de meilleure qualité. Maintenant que je suis ici, je vois la réalité : l'hôpital, ce n'est pas un lieu pour les Miskines (les pauvres, dans la langue arabe locale). Il faut encore beaucoup d'argent pour s'y faire soigner. D'ici l'hôpital est très loin donc c'est mieux d'y aller en bus, mais par manque 
de sous, il faut y aller à pied. Une fois arrivé, il faut acheter un carnet de santé pour la consultation. Si vous connaissez quelquiun ou avez de l'akalgoro (de l'argent pour un pourboire), vous pouvez facilement éviter d'attendre. La séance avec le médecin dépend de son humeur... La plupart du temps, c'est un gâachis de temps et d'argent, et le traitement est de mauvaise qualité. Avec les choukous (vendeurs ambulants des médicaments et de soins sans licence), il n'y a aucun problème: pour les maux de tête, on paie 25 FCFA, pour le paludisme 100 FCFA, et en plus ils sont serviables... Je ne crois pas que le traitement des choukous soit dangereux. C'est juste de la manvaise publicité : sil y a un cas d'empoisonnement ou de décès avec les choukous, ce n'est que la volonté d'Allah.

Bien que Diguel fasse partie intégrante de N'Djamena, la vie y est à la mode des campagnes. De jour en jour, les résidants déploient des efforts pour reconstruire leurs activités économiques, mais les possibilités sont limitées. L'environnement politique et socioéconomique n’offre aucune opportunité pour améliorer ses conditions de vie, et il laisse chez les individus un sentiment d'anxiété perpétuel qui affecte leur bien-être physique et psychologique. Une productivité insuffisante et instable provoque l'insécurité alimentaire. Puisque leur survie est fondée sur la culture et l'espace de vie, le dérangement de ces opportunités affecte profondément la dynamique des réseaux sociaux, et crée des sentiments de dépression et de stress.

\section{Nguéli : un quartier de N'Djamena sur la route du Cameroun}

Nguéli est situé au sud-ouest de N'Djamena, au bord de la rivière Logone qui sépare le Tchad du Cameroun. Nguéli fait partie du $9^{\text {ème }}$ arrondissement de la ville de N’Djaména et est peuplé de différents groupes ethniques venant de divers horizons. Lors de la guerre civile au Tchad, de 1979 à 1982, de nombreuses maisons ont été soit détruites, soit occupées par l'armée ou les rebelles ; de nombreuses personnes ont fui au Cameroun. Certaines ont choisi de ne pas revenir, même après la fin des conflits. D'autres personnes souhaitaient vivre près de Kousseri, la ville camerounaise de l'autre côté du pont, qui est leur nouveau centre d'activité pour les activités de création de revenus et les réseaux sociaux. Ceci a provoqué l'expansion de Nguéli, qui s'est également développé à la suite de l'arrivée de milliers de réfugiés congolais en 2003. Nguéli est le lieu de transfert de marchandises entre le Nigeria, le Cameroun et le Tchad ; c'est également un lieu où de nombreux militaires passent leur temps. Cela n'a pas été facile pour notre équipe de recherche d'accéder à ce quartier en raison du manque de confiance de la population et des dangers liés à un environnement potentiellement criminel. Heureusement, avec l'aide de Fottor Moussa Melé, le fils de l'ancien chef de Baro, nous avons pu gagner la confiance de la communauté Hadjaray, surtout les Migami de Nguéli.

Nguéli semble chaotique, et il est difficile d'établir les frontières entre chaque maison. Il n'existe pas de chemin ; les habitants marchent entre les habitations d'argile pour retrouver leurs propres foyers, pour rejoindre la rivière ainsi que la grande avenue qui lie Nguéli et le pont. Il n'existe pas de latrines ou d'eau propre, ou encore de système de drainage. Comme pour Diguel, les groupes d'Hadjaray vivent ensemble. Nous avons travaillé avec 30 ménages Migami, dont 12 étaient dirigés par des femmes : 5\% sont venus s'installer à N'Djamena avant 1980, 83\% entre 1980 et 1990 et $12 \%$ entre 1990 et 2003 . La majeure partie d'entre eux a quitté la région de Guera entre 1980 et 1990 en raison de la famine, de l'insécurité et de la répression politique. 
Le district de Nguéli offre des opportunités économiques pour les activités commerciales illégales et le travail légitime (jardinage, culture de riz, travail domestique, taxis-moto, blanchisserie) à N'Djamena et Kousseri. La plupart des femmes marchent dans les quartiers pour ramasser des feuilles de savonnier, qui constituent la base des sauces dans la cuisine Hadjaray : cela leur donne le sentiment qu'elles n’ont pas quitté le Guera. Nguéli est équipé de centres sanitaires et d'écoles dans le voisinage, mais selon les habitants et nos propres observations, l'accès à ces ressources dépend du régime politique en place et des manœuvres sociales. La situation à Nguéli est, à cet égard, très différente de celle de Diguel, où la politique se limite au logement. Lorsque Hissein Habré est venu au pouvoir en 1982, l'armée a envahi les structures sociales et économiques de Nguéli. La situation s'est depuis considérablement détériorée : le trafic de marchandises est dangereux, et l'armée abuse et torture constamment. Vingt-cinq des femmes dans notre échantillon ont confirmé avoir participé à ce type d'activité commerciale, et à avoir depuis perdu leur capital financier.

Les services de laverie sont une autre opportunité d'emploi pour les femmes à N'Djamena et Kousseri. Les femmes sont payées soit en espèces, soit en nature (savon, sucre, thé, vêtements) et reçoivent également le son des céréales qui leur a permis, pour certaines d'entre elles, de mettre sur pieds des petites entreprises. Le son des céréales est en effet souvent utilisé pour produire l'alcool traditionnel distillé (et interdit) que l'on appelle Argui, qui se vend bien. Depuis 1992, avec la venue des machines à décortiquer, ce travail n'est plus lucratif pour les femmes Hadjaray.

Les réunions de groupe à Nguéli sont impossibles en raison de l'omniprésence de l'armée, qui soupçonne que tout transport de marchandises est frauduleux. Les personnes impliquées dans ces activités peuvent ainsi facilement rencontrer des problèmes. Kaltouma décrit la manière dont l'armée traite les habitants de Nguéli :

On vous autorise à transporter des choses de N'Djamena à Nguéli, mais les militaires considèrent tout ce qui est transporté de Nguéli à la ville comme trafic clandestin. Il y a des karankaran et de bogo-bogo II partout; ils peuvent vous frapper jusqu'à la mort. Regardez les cicatrices que jai sur mon épaule droite. Ce sont les douaniers mobiles qui en sont responsables. L'année dernière, je me rendais à Mardjandafak, où ma sour allait donner naissance. J'avais du savon et des vêtements dans mon sac. Le douanier mobile m'a arrêté au niveau du pont. Alors que j'ai tenté de m'expliquer, l'un d'entre eux m'a frappé à l'épaule, en me traitant de menteur. Ils ont pris mon sac et tout son contenu. C'est ce qu'ils font à tout le monde. Si quelqu'un tente de senfuir ou de résister, ils peuvent facilement utiliser un revolver et le tuer. Je suis rentrée difficilement à la maison ce jour-là ... ils sont à la fois chefs et avocats, alors que peut-on faire, en vérité? (Kaltouma, Nguél, janvier 2005)

Le jardinage reste l'unique activité économique certaine, car elle génère des revenus et donne aux femmes la possibilité de se concentrer sur le commerce des légumes. Cependant, même avec le jardinage, il existe de nombreux conflits, et la gestion de ce secteur est devenue difficile. Certaines personnes utilisent leurs réseaux politiques pour s'approprier les terrains, mais l'armée a déjà pris possession des terres fertiles le long de la rivière, et les soldats n'hésitent pas à manipuler et à user de violence pour atteindre leurs objectifs. 
Les femmes avec lesquelles nous avons travaillé ont indiqué que la vie était devenue chère et plus difficile. Il y a18 ménages dont le logement est garanti et ceux qui sont arrivés avant 1980 ont eu la possibilité d'acheter un terrain, bien que ce type de transaction soit trop cher aujourd'hui. Les ménages comprennent en moyenne 4,5 individus, et le partage des pièces est chose commune. L'alcoolisme et le libertinage sont de plus en plus fréquents parmi les hommes de la communauté, et les femmes préfèrent divorcer de leur mari plutôt que de risquer d'attraper le SIDA, qui est lui aussi de plus en plus présent dans la région.

Les adolescents sont employés dans le secteur du transport et le monde de la moto en qualité d'apprentis et de mécaniciens. Dans certains cas, ces activités sont bénéfiques pour la famille, mais souvent les jeunes commencent à vivre dans la rue (De Bruijn et Djindil, 2006). Les filles se marient très jeune, ou deviennent prostituées. Les grossesses hors mariages sont fréquentes, et cela a un impact considérable sur le bien-être des ménages. Les cas de maladies sexuellement transmissibles sont de plus en plus fréquents parmi les jeunes, ainsi que les grossesses précoces, ce qui accentue la mortalité maternelle et infantile. Un enfant sur trois meurt avant d'atteindre l'âge de cinq ans. L'âge moyen des femmes pour leur première grossesse n'est que de 13,5 ans.

Parmi les 40 enfants âgés de six à 59 mois, 24 souffrent de malnutrition aigue, 16 connaissent un retard de croissance. 20 ont été complètement immunisés en raison de la proximité du centre médical et de la motivation des mères elles-mêmes. Parmi les mères, dix-huit ont un déficit pondéral parmi lesquelles neuf sous une forme sévère avec des IMC inférieurs à $16 \mathrm{~kg} / \mathrm{m}^{2}$. Les taux d'hémoglobine ont montré que 25 mères sont anémiées, avec un taux $\mathrm{Hb}$ de moins de $12 \mathrm{~g} / \mathrm{dL}$, et que 13 d'entre elles en souffrent sévèrement. Leur consommation de protéines animales est assez élevée et 20 des ménages n'ont que deux repas par jour. Ces résultats ne correspondent pas avec l'état nutritionnel des mères et des enfants, ainsi qu'avec l'anémie des mères.

Nous nous sommes ainsi posé des questions quant à la qualité de ces protéines consommées régulièrement. Le marché national du bétail a lieu deux fois par semaine à Nguéli. Tous les animaux qui ne peuvent pas traverser le pont pour cause de maladie sont abattus, et leur viande est vendue sur les marchés locaux. Beaucoup d'habitants de N'Djamena viennent souvent acheter de la viande bon marché à Nguéli. Le soir, lorsque la viande commence à pourrir, les prix chutent et ceci offre l'opportunité aux pauvres de s'en procurer. Les ménages dont l'un des membres travaille pour un boucher reçoit de la viande gratuitement. C'est le cas des dix ménages enquêtés. L'eau consommée vient des puits et de la rivière, avec tous les problèmes de contamination que cela implique. C'est la seule source d'eau accessible à la majorité pour le lavage, la boisson et la cuisine. Treize ménages dans notre étude achètent de l'eau du robinet (20 litres pour 50 FCFA). Il n'existe aucun système municipal de ramassage des déchets ou d'évacuation des eaux usées ; les maisons sont proches les unes des autres, et seuls les habitants connaissent les limites de leurs concessions. La nuit, ils utilisent des bougies pour l'éclairage.

Ces piètres conditions de vie donnent lieu à des risques et à de forts niveaux de violence, ce qui rend une vie normale impossible. Une pression constante est exercée par les agents publics (certains le sont vraiment, certains mentent sur leur véritable profession), qui ont le droit de pénétrer dans les propriétés pour chercher des preuves d'activités frauduleuses et pour menacer les familles qui y vivent. Après avoir fait face à des officiers des douanes qui ont détruit tous 
leurs biens, 22 des femmes dans notre étude ont abandonné le commerce des biens manufacturés, et se sont limitées à des petits commerces de vente de produits locaux dans leur propre cercle social. Cela leur permet de gagner en moyenne 650 FCFA par semaine, ce qui ne permet même pas de satisfaire les besoins quotidiens d'une personne. Pour s'entraider, les femmes organisent des tontines, auxquelles elles contribuent à hauteur de 200 FCFA par semaine, somme qui leur sera restituée plus tard. Ce système a également été touché par la détérioration des conditions socioéconomiques. Dans le passé, les femmes parvenaient à économiser 300 FCFA par semaine, mais beaucoup d'entre elles sont désormais incapables de gagner un tel montant ; elles ont donc décidé de réduire leur contribution, afin de donner la possibilité aux plus pauvres de commencer ou de maintenir une petite entreprise. C'est le cas de Khamissa, dont le mari semble physiquement en bonne santé, mais qui souffre de troubles psychologiques. Durant la guerre, il était soldat dans l'armée d'Hissein Habré, mais cela a fait de lui une personne moralement corrompue et antisociale. Les membres de la communauté savent qu'il a commis des atrocités sur les membres de leurs propres familles : il n'est le bienvenu ni parmi les migrants Migami, ni dans son propre village. Il incombe donc à sa femme Khamissa de faire son possible pour assurer la survie de leur famille. Khamissa était la plus pauvre parmi les femmes de la communauté, et pour des raisons financières, elle a marié sa fille de douze ans à un homme de soixante ans qui a déjà deux femmes. Elle s'explique :

Je suis mère de trois enfants. Le commerce de charbon que je fais à la maison est l'entreprise la plus sure que je puisse assurer aujourd'hui. Je dois faire attention et maintenir mon minimum. C'est mon capital vital, et si je vends du sucre ou du savon, je risque de tout perdre. Je n'ose rien vendre sur le bord de l'autoroute, bien que j'aurais beaucoup plus de clients. Le Conseil municipal me facturerait le droit de la place, et les douaniers n'hésiteraient pas à confisquer mon charbon. Tous mes voisins savent que je vends du charbon ici ; je gagne environ 300 FCFA par jour, parfois plus, selon mes clients et ce dont ils peuvent se permettre d'acheter.. J'ai une grande seeur qui a un bon emploi à N'Djamena, mais je ne peux pas lui demander de l'aide pour des raisons personnelles. Je n'ai pas non plus de contact avec ma famille au village.

Nguéli affecte négativement toutes les sources de revenus de ses habitants. Il existe des opportunités, mais il y a également des obstacles qui gênent les efforts de chacun. Le stress quotidien, la violence et l'insécurité réduisent la force morale et physique des habitants et rendent les ménages vulnérables à tous les niveaux. Les possibilités d'obtention de la nourriture sont hypothétiques et risquées. La pression constante exercée par les militaires opprime toutes les initiatives des habitants, dégrade leur moral et amplifie les sentiments d'incertitude quant au futur. De la même manière, le manque de confiance et l'insécurité limitent tout développement social et économique.

\section{Secteur 4 : Mongo}

La situation dans les zones périphériques de N'Djamena n'est pas unique au Tchad, et les populations des villes plus petites à l'intérieur du pays connaissent des problèmes identiques, comme l'illustre l'exemple de Mongo.

Mongo est la capitale de la province de Guera, dans la région centre du Tchad. Il s'agit de la cinquième plus grande ville du pays, et sa population est estimée à 27000 habitants $^{\mathrm{III}}$, dont $98 \%$ sont musulmans. La ville est un centre administratif, où les quelques organisations inter- 
nationales actives dans la région ont leurs bureaux et où divers services gouvernementaux, hôpitaux et l'école secondaire se trouvent - dans de vieux bâtiments qui rappellent à la ville son passé colonial. Mongo abrite un important marché régional le mercredi. Malgré ses magasins, son marché au bétail, sa mission catholique, sa mission protestante, ses deux grandes mosquées et bien d'autres plus petites, la ville est, en apparence et en réalité, pauvre. Cependant, les niveaux de pauvreté varient d'un quartier à l'autre.

La ville a attiré des familles et individus qui ont fui leurs villages en raison de la pauvreté, de la violence liée à la guerre et aux troubles civils, de la sécheresse et des mauvaises récoltes (De Bruijn et al., 2004). La ville a toujours été la base de réservistes de l'armée, mais en raison de la crise du Darfour, le gouvernement y a stratégiquement déployé des milliers de jeunes soldats (UNICEF, 2007). Par conséquent, nous avons trouvé de nombreux enfants soldats dans les rues de Mongo en 2007/2008. C'est une situation qui inquiète la population locale, et attire même les médias internationaux. Mongo n'est pas seulement un refuge pour les individus dans le besoin ou ceux qui cherchent un emploi, elle est également sollicitée à cause du centre médical provincial.

Durant les sécheresses des années 1980, de nombreux ruraux se sont réfugiés à Mongo car une aide humanitaire y était disponible. Pour organiser la distribution de nourriture à ce moment, la ville avait été divisée en neuf secteurs. Le Secteur 4, où nous avons effectué nos recherches, se situe en bordure de Mongo, et porte jusque là ce nom. Il s'agit du quartier le plus pauvre de Mongo, et ses habitants sont pour la plupart des migrants « de la crise ».

Au premier abord, le Secteur 4 ressemble à un village poussiéreux désorganisé. Les habitations sont des huttes éparpillées au pied d'une montagne. L'équipement des ménages dans les huttes est minime : il n'y a pas de latrines, et l'eau vient des puits en dehors de Mongo. Les petits chemins qu'utilisent les habitants pour se rendre en brousse constituent la seule forme d'infrastructure permanente. Ce secteur ne dispose pas d'école ni de centre de santé. Les maisons de meilleure qualité appartiennent soit aux fonctionnaires, soit aux veuves des riches commerçants.

Il semble qu'avant 1979, cette partie de Mongo était un espace vert avec de nombreux arbres. Cependant, les soldats de l'armée régulière les ont détruits. En effet, lorsque la guerre civile a commencé en 1979, les dirigeants rebelles de l'époque, le CDR, ont établi leur quartier général à la mission protestante de Barbassa, qui est proche du Secteur 4. Pour avoir une vue dégagée des itinéraires utilisés par les rebelles, ils ont coupé tous les arbres alentour (De Bruijn, 2006), les hommes ont été sauvagement assassinés ou pendus par les rebelles; les femmes et les enfants ont été abusés, torturés et leurs propriétés leurs ont été volées. Après la chute du CDR, la zone est devenue un espace libre. Durant les sécheresses des années 1980, les villageois se sont installés là pour recevoir de la nourriture et des soins. Plus tard, d'autres personnes des villages les y ont rejoints. D’autres qui ont emménagé au Secteur 4 avait déjà vécu dans les quartiers un peu plus riches de Mongo, mais ont dû vendre leurs maisons pour payer leurs factures médicales et nourrir leurs familles.

Le Secteur 4 est devenu un refuge pour les démunis et la majeure partie d'entre eux sont désormais considérés comme désavantagés (veuves, non-voyants, lépreux, etc.). Les dates auxquelles 
les 48 ménages de notre échantillon se sont installés dans le Secteur 4 sont les suivantes : 14\% entre 1960 et 1979, 59\% entre 1980 et 1990 et $27 \%$ entre 1991 et 2003 . Parmi les 48 ménages, 42 sont dirigés par des femmes (dont 24 veuves et 18 divorcées).

Nous avons reconstitué l'historique de l'occupation de la zone avec les informations obtenues lors de nos entretiens. Sur les 48 ménages de notre échantillon, 24 sont arrivés au Secteur 4 au moment d'une sécheresse, 11 s'y sont installés en raison de divers problèmes (sorcellerie, vol, viol, adultère), huit en raison de la guerre et des activités des rebelles. Cinq des femmes se sont installées dans le quartier sans raison particulière - mais la pression économique et sociales y sont pour quelque chose. L'historique de vie de la plupart de ces personnes révèle de multiples raisons quant à leur présence dans le Secteur 4, et au fait qu'elles y sont restées. La sécheresse est liée à la guerre, et l'une des femmes nous a indiqué de manière explicite : "si j'étais restée dans mon village, je serais morte de famine comme les autres ». Les résultats de cette famine ont été de plus catastrophiques en raison de la longue guerre civile. Cinq des résidentes, trois femmes âgées et deux femmes d'environ 40 ans, nous ont fait part de l'histoire du Camp IDP établi à Barbaza/Secteur 4 dans les années 1980, lorsque la province de Guera a connu de nombreux problèmes après la montée des violences et la sécheresse. A cause de la guerre, l'infrastructure du pays était de très mauvaise qualité, et les ONG n'avaient que peu ou pas accès aux personnes qui vivaient dans les zones rurales. La Croix-Rouge et le Programme Alimentaire Mondial ont été les premières organisations humanitaires internationales à arriver à Mongo et à aider la population locale - parmi elle, les femmes citées ci-dessus, qui se voient comme "réfugiées ». La Croix-Rouge n'est pas restée longtemps dans la région, mais après son départ, les individus que l'organisation avait attirés à Mongo ne sont pas repartis du Secteur 4. L'une des femmes nous a confié qu'elle pouvait y acheter un terrain pour 1500 FCFA, et une autre nous a indiqué que les terres sont disponibles dans cette partie de la ville, considérée comme rurale.

La malnutrition est presque endémique dans le Secteur 4. Les enfants naissent et grandissent dans des conditions de famine; le taux de mortalité infantile est de $47 \%$. Sur 42 enfants âgés de 6 à 59 mois, 40 souffrent de malnutrition aiguë, 38 d'entre eux souffrent de malnutrition chronique. La santé de leur mère est également mauvaise : leur IMC moyen est de $16,7 \mathrm{~kg} / \mathrm{m}^{2}$, ce qui se situe en dessous de l'IMC normal, qui est de $18,5 \mathrm{~kg} / \mathrm{m}^{2}$. Nous ne pouvons nous prononcer sur la fréquence des repas dans les ménages, car il est très difficile d'accéder à la nourriture. Les repas sont généralement composés de sorgho accompagné de différentes sauces ; les locustes sont la principale source de protéines pour tous les ménages. Aucun des ménages ne s'attend à jouir de la sécurité alimentaire, et c'est pour eux une bataille constante pour acquérir des aliments. Lorsque nous avons mené nos entretiens, personne n'avait de céréales en stock. Il s'agit là d'un contexte de pauvreté endémique où l'espoir est absent. La santé et l'éducation ne sont pas des priorités pour $89 \%$ des ménages, et d'autres considèrent que ces services sont destinés " à ceux qui sont riches et qui mangent bien ".

Tous les membres des ménages du Secteur 4 contribuent à la recherche quotidienne d'aliments, dont les enfants, les infirmes et les handicapés qui mendient près de la mosquée et des marchés. Personne dispose d'une source stable de revenus. Dans 30 des ménages, au moins l'un des membres mendie. Pour les habitants du Secteur 4, la mendicité est un important aspect de la 
vie quotidienne. Les vieilles femmes, dont certaines sont aveugles, mendient le vendredi. D'autres mendient le dimanche à la sortie des églises. Ces femmes errent dans la ville durant la semaine, se rendent au marché et quémandent de maison en maison, et reçoivent ainsi une importante partie de la nourriture destinée à leur famille auprès d'autres personnes. Ces personnes ont été heureuses de nous montrer les recettes de leurs activités. Les fêtes religieuses, les prières du vendredi et les diverses cérémonies (mariages, funérailles, baptêmes et circoncision) offrent la possibilité de manger de la viande et d'avoir des repas.

Tous considèrent leur vie difficile, mais l'acceptent : " la vie au village n'est-elle pas pire ? . Beaucoup ont des problèmes de santé mais n’ont pas les moyens de s'offrir un traitement médical. Ils doivent tous s'occuper de leurs enfants, soit les leurs, soit ceux de leurs enfants décédés ou ceux qui ont émigré. L'avantage de vivre dans cette zone est que les terrains sont libres, et qu'il existe des réseaux sociaux. L'agriculture est l'unique activité qui peut générer des revenus pour les ménages, mais le sol de mauvaise qualité doit être fertilisé, ce qui décourage bon nombre d'individus. Même si la vie est difficile, les habitants trouvent le moyen de survivre. L'histoire de leur vie montre la manière dont ils ont réagi aux difficultés qu'ils ont rencontrées :

Il y a trois ans, j'ai arrêté de travailler sur mon propre champ : avec l'estomac vide, je n'avais pas la force nécessaire. J'ai donc décidé de chercher de la nourriture dans la campagne alentour, en espérant trouver quelque chose pour nourrir ma famille, mais je n'ai rien trouvé de satisfaisant. C'est toujours un échec. De nombreux individus au Secteur 4 préferent mendier ou travailler pour quelqu'un en ville. Si vous êtes aveugle, ou si vous avez la lèpre, la mendicité est une solution qui marche. Les gens peuvent vous donner quelque chose, par pitié. Le travail manuel est également une bonne solution, mais beaucoup de personnes à Mongo paient en fonction de leur récolte, donc le salaire n'est jamais fixe. Je préfere le liquide. C'est aussi difficile de trouver ce type de travail. Heureusement, l'année dernière, j'avais travaillé dans les champs des Arabes, et cela m'a permis de gagner 20 plants de sorgho rouge et 5000 FCFA. J'espère trouver un travail similaire cette année. Qui sait ce qui va se passer dans le futur? Au Tchad, les lendemains n'appartiennent à personne.

Bozile Soumaine, 55 ans, Secteur 4, octobre 2005

Les habitants sont contraints de vivre dans la misère en raison de diverses formes de violence (physique ou morale). Au niveau administratif, à Mongo, on ne prête aucune attention à la situation, bien que certaines organisations privées offrent une assistance dans des cas individuels. La Mission Protestante est un excellent exemple de ce type d'assistance, qui s'occupe des lépreux et des enfants aveugles. Certaines organisations musulmanes offrent également leur assistance, et les résidents se convertissent de plus en plus à l'Islam pour bénéficier de cette assistance, et pour réduire le fardeau de leurs souffrances. Le Secteur 4 est l'exemple typique de l'exclusion sociale : c'est un lieu où les laissés-pour-compte et les malchanceux des villages et de la ville sont concentrés. L'agriculture pourrait être la méthode idéale de garantir la sécurité alimentaire, mais les terrains sont épuisés.

Les réseaux d'assistance peuvent être fondés sur un soutien matériel ainsi que sur un support moral. Comme Platteau (1991) l'a indiqué, un réseau de soutien sans richesse matérielle à sa 
base est condamné à échouer. Platteau introduit l'idée d'une covariance du risque : nos données montrent cependant que le soutien moral en soi peut apporter une différence dans la survie des individus. Les entretiens et les observations montrent que les voisins dans cette zone tentent en effet de s'entraider des deux manières. Les réseaux de soutien impliquent souvent des voisins proches, mais sont généralement composés d'enfants ou de membres de la famille. Les individus partagent les mêmes vies, et confier ses problèmes aide à rendre le quotidien plus supportable. De telles relations peuvent également se former au cas par cas au fur et à mesure que de nouveaux habitants arrivent, et que d'autres déménagent. L'existence de ces réseaux nous est apparue clairement lorsqu'un des habitants est décédé : toutes les femmes se sont rendues chez cette personne ; de même, un bébé est né, et tous ont rendu visite au nouveau-né avec de petits cadeaux. Dans ce sens, le Secteur 4 est devenu un voisinage social, et cet aspect n'est pas essentiellement lié à l'ethnicité ou aux relations dont les individus jouissaient lorsqu'ils habitaient au village, mais plutôt au fait que ces personnes partagent des conditions de vie identiques et font bien entendu partie de la même famille. Dans un autre cas, une femme vit avec son frère, qui est propriétaire d'une des grandes exploitations agricoles. Son histoire montre cependant que prendre soin d'une autre personne est une situation par nature limitée, et qu'il incombe en fin de compte à l'individu lui-même de prendre soin de lui ou elle.

Le tableau suivant résume certains des résultats des trois zones étudiées.

Tableau 1 : caractéristiques socio-démographiques, nutritionnelles et sanitaires des trois zones étudiées

\begin{tabular}{|c|c|c|c|}
\hline & \multicolumn{2}{|c|}{ N'Djamena } & \multirow{2}{*}{$\begin{array}{c}\text { Mongo } \\
\text { Secteur } 4 \\
\mathrm{~N}_{\mathrm{h}}=48 / \mathrm{Nc}=42\end{array}$} \\
\hline & $\begin{array}{c}\text { Diguel } \\
\mathrm{N}_{\mathrm{h}}=33 / \mathrm{Nc}=36\end{array}$ & $\begin{array}{c}\text { Nguéli } \\
\mathrm{N}_{\mathrm{h}}=30 / \mathrm{Nc}=40\end{array}$ & \\
\hline $\begin{array}{l}\text { Caractéristiques des ménages } \\
\text { Pourcentage des membres des ménages } \\
\text { éduqués } \\
\text { Pourcentage des femmes chef de famille } \\
\text { Moyenne d'âge des dépendants } \\
\text { Age moyen du chef de famille }\end{array}$ & $\begin{array}{c}18 \\
45 \\
4 \pm 1.5 \\
26 \pm 7\end{array}$ & $\begin{array}{c}36 \\
40 \\
4.5 \pm 1.6 \\
29 \pm 8\end{array}$ & $\begin{array}{l}20 \\
87 \\
3.2 \pm 1.4 \\
33 \pm 12\end{array}$ \\
\hline $\begin{array}{l}\text { Etat sanitaire et nutritionnel } \\
\text { Pourcentage des enfants souffrant de retards } \\
\text { de croissance } \\
\text { Pourcentage des enfants chétifs } \\
\text { Taux de mortalité infantile } \\
\text { Pourcentage des enfants immunisés } \\
\text { Valeur moyenne de l'hémoglobine des } \\
\text { mères } \\
\text { Valeur moyenne de l'IMC des mères }\end{array}$ & $\begin{array}{c}61 \\
47 \\
40 \\
19 \\
9.97 \pm 1.64 \\
17.02 \pm 2.32\end{array}$ & $\begin{array}{c}60 \\
40 \\
33 \\
44 \\
11.14 \pm 1.50 \\
18.06 \pm 2.70\end{array}$ & $\begin{array}{c}95 \\
90 \\
64 \\
\text { None } \\
\text { nd } \\
16.70 \pm 2.80\end{array}$ \\
\hline $\begin{array}{l}\text { Conditions de vie } \\
\text { Pourcentage des ménages disposant d'eau } \\
\text { potable } \\
\text { Accès aux soins médicaux et à l'éducation } \\
\text { Emploi sur } \\
\text { Crainte de perdre son terrain/son logement } \\
\text { Pression psychologique } \\
\text { Pourcentage des ménages souffrant } \\
\text { d'insécurité alimentaire } \\
\text { Nombre de repas quotidiens }\end{array}$ & $\begin{array}{c}100 \text { (Temporary) } \\
3 \\
18 \\
74 \\
\text { all } \\
72 \\
1,3\end{array}$ & $\begin{array}{c}43 \\
6 \\
12 \\
60 \\
\text { all } \\
67 \\
1,9\end{array}$ & $\begin{array}{l}\text { None } \\
\text { None } \\
\text { None } \\
\text { None } \\
\text { all } \\
\text { all } \\
\text { nd }\end{array}$ \\
\hline
\end{tabular}

$N b=$ Nombre de ménages, $N c=$ nombre d'enfants en dessous de cinq ans 


\section{Discussion}

Que peut-on apprendre des cas présentés ci-dessus ? L'image que nous dressons dans la présente contribution illustre la manière dont les individus survivent dans les districts urbains du Tchad, et révèle les vies des victimes négligées des crises humanitaires. Pour comprendre la vie quotidienne de ces personnes et la base de leur organisation sociale, nous avons eu besoin d'informations concernant l'histoire et les facteurs actuels qui influencent leurs réseaux socioéconomiques, leur intégrité morale et physique ainsi que la manière dont ces éléments ont contribué aux obstacles qui les empêchent de gagner leur vie. Nous avons combiné des méthodes qualitatives et quantitatives pour tenter d'établir un portrait des conditions de vie et des activités économiques des ménages et des individus dans ces communautés

Nos données qualitatives et quantitatives nous ont permis de mieux comprendre l'interaction entre les individus et leur contexte social. Comme Sen (1983) l'indique, « la capacité d'un individu à contrôler - et en fait, à contrôler toute commodité qu'il souhaite acquérir ou garder - dépend des relations de droit qui gouvernent les aspects de la possession et de l'utilisation dans la société concernée. Cela dépend de ce que l'individu possède, du type de possibilités d'échange qui lui sont offertes, ce qui peut lui être donné gratuitement, et ce qui est pris de lui ». Tout trouble à ce niveau est fatal pour la stabilité de la société et des ménages : la dynamique même de la société peut être touchée. L'émergence de tensions, de violences, de restrictions ou un déclin dans les relations sociales, comme nous avons pu l'observer dans ces quartiers, constituent une réaction à ce niveau de trouble. La pression des pouvoirs publics, de la violence structurelle et de l'inégalité à N'Djamena ne permet pas à ces migrants à Diguel, et surtout à Nguéli, d'accéder aux opportunités de survie qui peuvent leur être disponibles, mais qui restent inaccessibles. Toutes les formes de négociations que ces individus adoptent dépendent d'un certain niveau de pouvoir de négociation, et dans ces contextes fortement sociaux mais fondamentalement pauvres, ce pouvoir constitue l'équivalent du pouvoir d'achat dans les environnements ou les ressources monétaires sont davantage disponibles. Parfois, ces personnes doivent faire des sacrifices, et, afin de survivre, certains migrants adoptent des habitudes et des comportements qui sont essentiellement opposés ou du moins peu cohérents par rapport à leurs normes et valeurs (la religion, par exemple). Ces personnes vivent par conséquent dans une peur constante de se faire rejeter par leurs propres communautés. De la même manière, pour obtenir certains emplois (comme garde, par exemple), les individus peuvent se voir forcés de se convertir à l'Islam par leurs employeurs. Le cas des adolescents qui se lancent dans la prostitution ou rejoignent des réseaux criminels pour aider leurs familles est un autre exemple. Ces changements de comportement imposés sont une réaction à de forts niveaux d'insécurité, et illustrent le fait que « la sécurité alimentaire, qui intègre la capacité personnelle d'acquérir de la nourriture appropriée par des moyens socialement acceptables, est limitée voire incertaine » (Allen, 1999; voir également Radimer et al., 1992).

Les données quantitatives nous ont permis de comprendre l'expression physique des difficultés rencontrées individuellement ou collectivement (par exemple au niveau des ménages). De telles données comprennent des informations sur l'état nutritionnel et la santé des mères et des enfants de moins de cinq ans ainsi que sur les taux de mortalité infantile. Dans les trois quartiers, les taux de malnutrition et de mortalité infantile trahissent la présence d'une crise humanitaire. 
La combinaison d'ingestion d'aliments inappropriés et en quantité insuffisante et de maladies altère la capacité physique et physiologique des individus, et provoque de graves conséquences sur le bien-être de leurs familles. Comme certaines études l'ont montré, la malnutrition est une importante cause de maladies cérébrales, de faible poids et de malformations à la naissance, ainsi que de mortalité : ces problèmes contribuent au sous-développement physique et cognitif, au handicap mental et à la susceptibilité à long terme aux maladies infectieuses (Finch et Crimmins, 2004). Ces conséquences affectent négativement les capacités physiques et le potentiel intellectuel d'un individu.

La plupart des individus avec qui nous nous sommes entretenus ont subi un traumatisme quelconque, et de nouvelles générations grandissent et vivent dans une société toujours marquée par la famine, la violence et les troubles sociaux.

\section{Conclusion}

Les situations difficiles que connaissent les populations qui vivent en marge des zones urbaines du Tchad nous ont fait nous demander si ces zones sont considérées comme une opportunité ou comme un refuge pour les groupes exclus socialement. Dans quelle mesure le choix de déménager dans un autre quartier de la ville fait-il partie des décisions prises par les individus dans leur propre vie ? Ceux-ci sont à la fois victimes et participants actifs dans les circonstances problématiques dans le cadre desquelles leurs vies se développent. La plupart des migrants à N'Djamena sont de pauvres Hadjaray qui vivent en groupes selon leur origine ethnique. Ces groupements sociaux protègent et encouragent un " microclimat " social qui recrée, dans une certaine mesure, la vie qu'ils connaissaient au village. Les relations sociales génèrent la capacité de négocier l'accès aux ressources, aux biens et aux réclamations, dont l'espace de vie, et sont un moyen de reconstituer une certaine identité culturelle. Ce type de regroupement est une stratégie de survie souvent utilisée par les individus démunis qui vivent dans l'insécurité et l'instabilité (De Bruijn et Van Dijk, 1995). Le fait que ces individus choisissent d'occuper les zones périphériques des villes leur donne la possibilité de construire et de renforcer des relations mutuelles et de générer des ressources qui seraient en d'autres circonstances indisponibles.

Les victimes des crises humanitaires au Tchad ont dans le passé été négligées à la fois par le gouvernement tchadien et par les organisations internationales. Certaines des interventions particulières offrant une assistance technique, médicale et alimentaire ont été d'une certaine aide pour les victimes immédiates de crises aiguës. Cependant, cette assistance n’a pas touché de manière égale tous les individus, ou n'est pas arrivée au bon moment, ou encore n'a pas atteint les personnes qui étaient le plus dans le besoin ou qui vivaient en dehors des zones prévues. Une fois qu'une situation de crise devient endémique et détachée de l'événement qui l'a déclenchée, les populations touchées sont fréquemment abandonnées, laissant les victimes qui vivent dans la crainte dans des situations de misère et de pauvreté extrême, comme on l'a vu à Mongo et son secteur 4 .

La crise du Darfour attire actuellement des groupes différents (internationaux) qui offrent une assistance humanitaire amenée aux camps de réfugiés dans la région est du Tchad, au départ de N'Djamena. Les convois d'aide doivent traverser des régions du pays où la vie est misérable, et où les individus connaissent des conditions relativement similaires à celles des camps. 
Ces personnes négligées, qui luttent pour leur survie dans des communautés installées au bord de la route, voient passer les convois humanitaires qui se précipitent vers l'est du Tchad : ils ne reçoivent aucune attention, et n'ont pas de pouvoir de revendication.

\section{Bibliographie}

ALLEN, P. 1999. Reweaving the food security safety net: Mediating entitlement and entrepreneurship. Agriculture and Human Values, 16(2):117-129.

AZEVEDO, M.J. 1998. Roots of Violence: A History of War in Chad. Amsterdam: Gordon \& Breach. CHAMBERS, R. et CONWAY, G. 1992. Sustainable rural livelihoods: Practical concepts for the 21st century. IDS Discussion Paper 296, Brighton: Institute of Development Studies

DE BRUIJN, M. 2007. Agency in and from the margins: Street children and youth in N'Djaména, Chad. (In De Bruijn, M., Van Dijk, R. \& Gewald, J.B. eds. Strength beyond Structure: Social and Historical Trajectories of Agency in Africa. Leiden: Brill. p. 263-285

DE BRUIJN, M. et DJINDIL, N.S. 2006. État Nutritionnel et histoire de vie des 'enfants de la rue' à N'Djamena. Psychopathologie africaine 33(2):183-211.

DE BRUIJN, M et VAN DIJK, H. 1995. Arid Ways: Cultural Understandings of Insecurity in Fulbe Society, Central Mali. Wageningen: CERES.

DE BRUIJN, M et VAN DIJK, H. 2007a. Chad. (In Mehler, A., Melber, H. \& Van Walraven, K. eds. Africa Yearbook: Politics, Economy and Society South of the Sahara in 2006. Leiden: Brill Academic Publishers. p. 215-223.)

DE BRUIJN, M et VAN DIJK, H. 2007b. The multiple experiences of civil war in the Guéra region of Chad, 1965-1990. Sociologus, 57(1):61-98.

DE BRUIJN, M et VAN DIJK, H. 2008. Chad. (In Mehler, A., Melber, H. \& Van Walraven, K. eds. Africa Yearbook: Politics, Economy and Society South of the Sahara in 2007. Leiden: Brill. p. 223-230.)

DE BRUIJN, M et VAN DIJK, R. 2007. Introduction. (In De Bruijn, M., Van Dijk, R. \& Gewald, J.B. eds. Strength beyond Structure: Social and Historical Trajectories of Agency in Africa. Leiden: Brill. p. 263-285.)

DEVEREUX, S. 2001. Livelihood insecurity and social protection: A re-emerging issue in rural development. Development Policy Review, 19(4):507-520.

DICKOW, H. 2005. Democrats without Democracy?: Attitudes and Opinions on Society, Religion and Politics in Chad. Byblos: Centre International des Sciences de l'Homme.

DJINDIL, N.S., TABO, S.N et MOGOTA, A.T. 2007. Who benefits from public social spending in Chad? A benefit incidence analysis. PEP PMMA Working Paper 2007-11. [Web:]http://ssrn.com/abstract=975219. [Date of access:] 8 June 2009.

ESSED, P.H., FRERKS, G. et SCHRIJVERS, J. 2005. Refugees and the Transformation of Societies, Agency, Policies, Ethics and Politics. New York/Oxford: Berghahn Books.

ERIKSSON, H. et HAGSTRÖMER, B. 2005. Chad: Towards Democratization or Petro-dictatorship? Uppsala: Nordic Africa Institute. 
FINCH C.E. et CRIMMINS, E.M. 2004. Inflamatory exposure and historical changes in human lifespans. Science, 305 (5691):1736-1739.

INTERNATIONAL CRISIS GROUP (ICG). 2006. Tchad : vers le retour de la Guerre? Africa Report 111, 01-06-2006. [Web:] http://www.unhcr.org/refworld/docid/44c77d614.html [Date of access:] 8 June 2009.

GALLAIS, J. 1975. Pasteurs et paysans du Gourma : la condition sahelienne. Paris : C.N.R.S. (18):1233-239.

MILES, W.F.S. 1995. Tragic tradeoffs: Democracy and security in Chad. The Journal of Modern African Studies, 33(1):53-65.

OECD see ORGANISATION FOR ECONOMIC CO-OPERATNION AND DEVELOPMENT

ORGANISATION FOR ECONOMIC CO-OPERATION AND DEVELOPMENT. 2007. Chad: Perspectives économiques en Afrique 2007. p. 181-195.

PLATTEAU, J.P. 1991. Traditional system of social security and hunger insurance: Past achievements and modern challenges. (In Ahmad, E., Drèze, J., Hills, J. \& Sen, A.K. eds. Social Security in Developing Countries. Oxford: Clarendon Press. p. 112-170.)

RADIMER. K.L., OLSON, C.M., GREENE, J.C., CAMPBELL, C.C., et HABICHT, J.P. 1992. Understanding hunger and developing indicators to assess it in women and children. The Journal of Nutrition Education, 24(1):36-45.

RIBOT, J.C. et PELUSO, N.L. 2003. A theory of access. Rural Sociology, 68(1):153-181.

SEN, A. 1983. Poverty and Famines: An Essay on Entitlement and Deprivation. Oxford: Oxford University Press.

\section{UNDP see UNITED NATIONS DEVELOPMENT PROGRAM}

\section{UNICEF see UNITED NATIONS CHILDREN'S FUND}

UNITED NATIONS CHILDREN'S FUND. 2007. Central African Republic signed child soldier reintegration agreement. Press note, 16 June 2007. [Web:] http://www.unicef.org/infobycountry/media_40015.html [Date of access:] 8 June 2009.

UNITED NATIONS DEVELOPMENT PROGRAM. 2000-2008. Human Development Reports from 2000-2008. [Web:] http://hdr.undp.org/en/reports. [Date of access:] 8 June 2009.

UNITED NATIONS: FOOD AND AGRICULTURE. 1996. World Food Summit - Rome Declaration on World Food Security and WFS Plan of Action. WFS document. Rome: FAO.

USAID. 2008. Chad: Complex Emergency. Situation Report 2. [Web:] http://www.usaid.gov/our_ work/humanitarian_assistance/disaster_assistance/countries/chad/template/fs_sr/fy2008/chad_ce_ sr02_06-27-2008.pdf. [Date of access:] 8 June 2009.

VAN DIJK, H. 2007. Briefing: Political deadlock in Chad. African Affairs, 106 (425): 697-703.

WGI see WORLDWIDE GOVERNANCE INDICATORS

WHO see WORLD HEALTH ORGANISATION

WORLD HEALTH ORGANISATION. 1995. Physical status: The use and interpretation of anthropometry. World Health Organisation, Technical Report Series.

WORLDWIDE GOVERNANCE INDICATORS. 2008. Country data report for Chad, 1996-2007. Governance Matters. [Web:] http://info.worldbank.org/governance/wgi/pdf/c211.pdf. [Date of access:] 8 June 2009. 
Note

I. Gombo name for Okra

II. Bogo-bogo: Customs officer in charge of combating fraud. Karan-Karan: refers to fortyforty the number plate of customs` vehicles. Both have the connotation of corrupt officials because they usually abuse the population

III. See the web site for this estimation: www.tageo.com/index-e-cd-cities-TD-lg-fr.htm 